\title{
Optics measurement and correction during beam acceleration in the Relativistic Heavy Ion Collider
}

\author{
C. Liu, A. Marusic, M. Minty
}

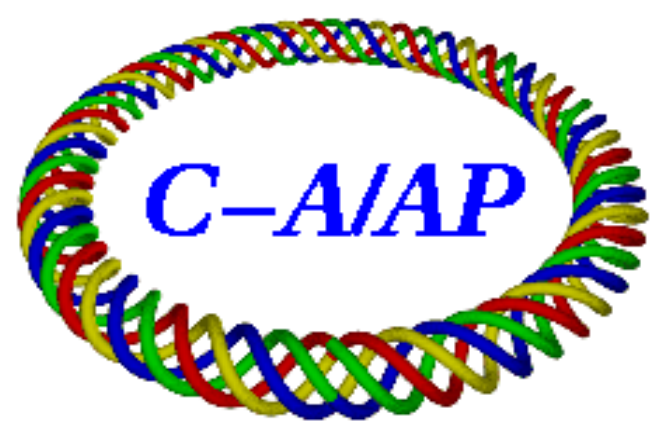

Collider-Accelerator Department Brookhaven National Laboratory Upton, NY 11973

\section{U.S. Department of Energy Office of Science, Office of Nuclear Physics}

Notice: This document has been authorized by employees of Brookhaven Science Associates, LLC under Contract No. DE-AC02-98CH10886 with the U.S. Department of Energy. The United States Government retains a nonexclusive, paid-up, irrevocable, world-wide license to publish or reproduce the published form of this document, or allow others to do so, for United States Government purposes. 


\title{
Optics Measurement and Correction during Beam Acceleration in the Relativistic Heavy Ion Collider
}

\author{
C. Liu, A. Marusic, M. Minty \\ Brookhaven National Lab, Upton, NY, U.S.A.
}

\begin{abstract}
To minimize operational complexities, setup of collisions in high energy circular colliders typically involves acceleration with near constant $\beta$-functions followed by application of strong focusing quadrupoles at the interaction points (IPs) for the final beta-squeeze. At the Relativistic Heavy Ion Collider (RHIC) beam acceleration and optics squeeze are performed simultaneously. In the past, beam optics correction at RHIC has taken place at injection and at final energy with some interpolation of corrections into the acceleration cycle. Recent measurements of the beam optics during acceleration and squeeze have evidenced significant beta-beats which if corrected could minimize undesirable emittance dilutions and maximize the spin polarization of polarized proton beams by avoidance of higher-order multipole fields sampled by particles within the bunch. In this report the methodology now operational at RHIC for beam optics corrections during acceleration with simultaneous beta-squeeze will be presented together with measurements which conclusively demonstrate the superior beam control. As a valuable by-product, the corrections have minimized the beta-beat at the profile monitors so reducing the dominant error in and providing more precise measurements of the evolution of the beam emittances during acceleration.
\end{abstract}

Keywords: Global coupling, dispersion, skew quadrupole, simultaneous correction, spin polarization, SVD

\section{Introduction}

The Relativistic Heavy Ion Collider (RHIC) is composed of two identical non-circular counter-rotating rings in a common horizontal plane, which are oriented to intersect with one another at six crossing points [1]. Each ring consists of three inner arcs and three outer arcs and six insertions joining the inner and outer arcs (Fig. 1).

The RHIC magnet ramping management system is flexible to allow betasqueeze on the acceleration ramp or independently at constant dipole field

Email address: cliu1@bnl.gov (C. Liu) 


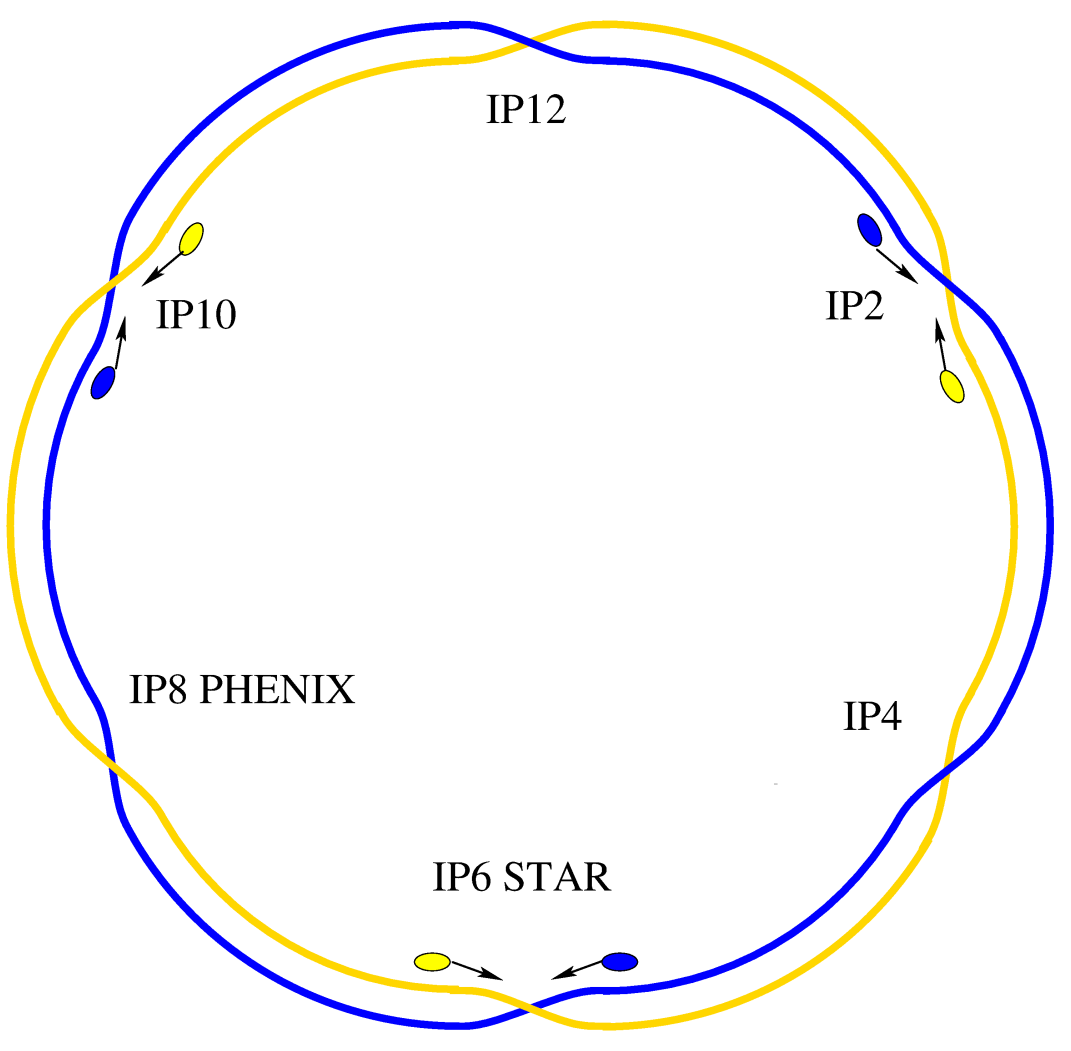

Figure 1: The schematics for the Relativistic Heavy Ion Collider [1]. 
by ramping insertion quadrupoles [2]. In RHIC, beam acceleration and betasqueeze are combined together for both polarized proton and heavy ion programs for mainly two reasons: it saves time by performing a simultaneous beam acceleration and beta-squeeze; and the insertion quadrupoles currents can be configured to be increasing on the way to top energy. Fig. 2 shows the beam rigidity and its derivative, the beta star values at the IPs on the ramp for $100 \mathrm{GeV} \mathrm{Au}-\mathrm{Au}$ physics program in 2014. Beta star at IP8 is the same as that for IP6, and beta stars at IP2, 4, 12 are the same as that for IP10. The magnet settings on a ramp are organized in time as a set of intermediate points, called step-stones, where the settings can be manipulated through an application called "RampEditor". The interpolation of the magnet strengths in-between step-stones is either linear or cubic splines, depending on the magnet type [3]. The machine optics data at step-stones can be accessed from on-line optics model by applications. Before feedback systems were implemented, the orbit, tune, coupling and chromaticity were tuned at step-stones to improve transmission efficiency on the ramp. Recently, all physics stores at RHIC are now established using simultaneous orbit, tune, coupling, and energy feedback during beam injection, acceleration to full beam energies, during beta- squeeze, and during removal of separation bumps to establish collisions [4]. Only manual tuning of chromaticities at step-stones is required for ramps in RHIC nowadays.

It is desirable to minimize the machine optics ( $\beta$-functions/phase advances) errors during beam acceleration to improve dynamic aperture for heavy ions and reduce depolarization resonance strengths for polarized proton program. However, it is not practical to pause at step-stones for optics measurement and correction in the simultaneous beam acceleration and beta-squeeze ramp. We demonstrated recently an on-the-fly beam optics measurement during beam acceleration and successfully implemented corrections which substantially suppressed beta-beats on the ramp in RHIC. The methodology of the measurement and correction will be presented in the following sections. In section 2, the beam optics measurement during beam acceleration will be introduced, which includes subsections on optics analysis based on interpolated Fast Fourier transform of turn-by-turn beam position monitor (BPM) data, acquiring turn-by-turn BPM data and optics analysis results during beam acceleration. In section 3 , beam optics correction will be presented, which includes optics corrections at a fixed beam energy and corrections during beam acceleration and setup for collision.

\section{Beam Optics Measurement During Beam Acceleration}

Turn-by-turn measurements of the beam position with an applied excitation to the beam has been used at many accelerators to infer fundamental optical parameters such as the tune, the phase advance between BPMs, and with input from the accelerator model, the $\beta$-functions. Many different algorithms for data analysis have been successfully applied such as fitting in time domain [5], interpolated FFT technique in frequency domain $[6,7,8]$ and statistical techniques (PCA, ICA) $[9,10]$ finding beam motions in a high dimension data. The 


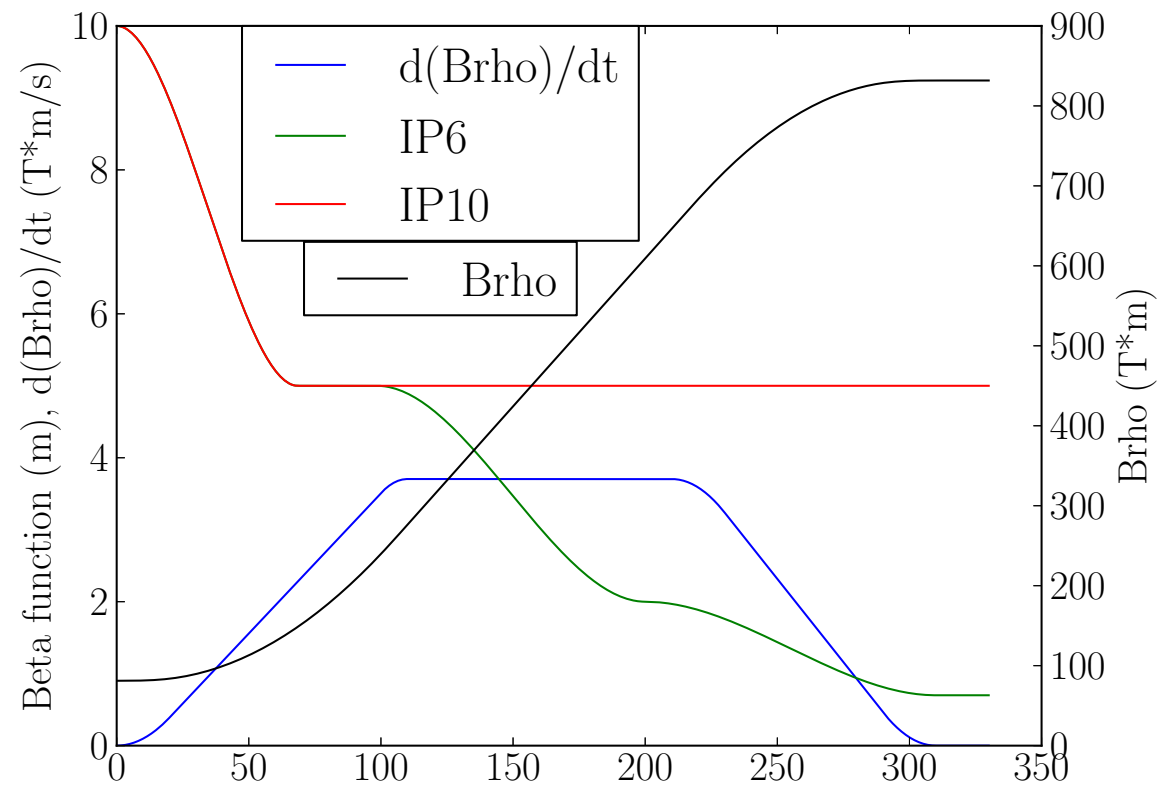

Figure 2: The beam rigidity and corresponding derivative, beta stars at IPs on the ramp for $100 \mathrm{GeV}$ Au physics program in 2014 . 
interpolated FFT technique was adopted in this report to analyze the machine optics in RHIC.

The acquired turn-by-turn BPM data with the beam kicked by the tune meter kicker multiple times usually oscillates for less than 500 turns because of decoherence for a typical chromaticity setting ( $\sim 2$ above transition, $\sim-2$ below transition). The application of interpolated FFT analysis on these BPM data yielded high precision tune, phase advances and $\beta$-functions measurement despite the limited data points acquired. This demonstration opens up the possibility of acquiring turn-by-turn BPM data with the tune meter on the ramp for optics measurement $[11,12]$.

\subsection{Optics Analysis Based on the Interpolated FFT of turn-by-turn BPM Data}

The analysis of the spectrum based on turn-by-turn BPM data can reveal the information of oscillation frequency which corresponds to the betatron tune, the oscillation amplitude which corresponds to the $\beta$-functions, and the phase of the oscillation which corresponds to the relative phase advances at the BPMs. With short-lived oscillation in the turn-by-turn BPM data, one needs to combat two effects, called energy leakage and the grid effect [13]. Energy leakage means energy leaks out of the main peak and produces sidebands, which in turn decreases the signal-to-noise ratio. Due to limited data points, the spectral intensities were only available at discrete frequencies. This means the real peak intensity lies in-between the discrete frequency lines, instead of at one of the spectral line with the highest intensity. This is so-called the grid effect.

By applying a window on the turn-by-turn BPM data, the energy leakage in the spectrum can be alleviated to some extent. The type of window depends on the specific application. A comprehensive comparison of different types of windows for high precision tune measurement was carried out in reference [14]. The Gaussian window was applied in our analysis for high precision measurement of both tune and phase advance. To further improve the precision, interpolation of the real peak in-between frequency lines was performed. A set of data points on the spectral peak was fitted as Gaussian distribution to determine the real peak position, which corresponds to the machine tune. A typical histogram of the measured tunes by applying interpolated FFT technique on a set of turn-by-turn data from $\sim 160$ BPMs is shown in Fig. 3.

The tunes retrieved from all BPMs were averaged. The averaged tune was applied in a continuous FFT for calculation of the phases [15].

$$
\begin{gathered}
X(f)=\frac{1}{N} \sum_{n=1}^{N} x_{n} \exp (-2 \pi f n i), \\
\phi=\operatorname{angle}(X)
\end{gathered}
$$

Where $N$ is the number of data points of the turn-by-turn BPM data. The phase calculated here is the relative phase. The calculated phases will be shifted by a constant offset to best match the model phase advances. The measured phases are largely model independent. The statistic error of the phase measurements 


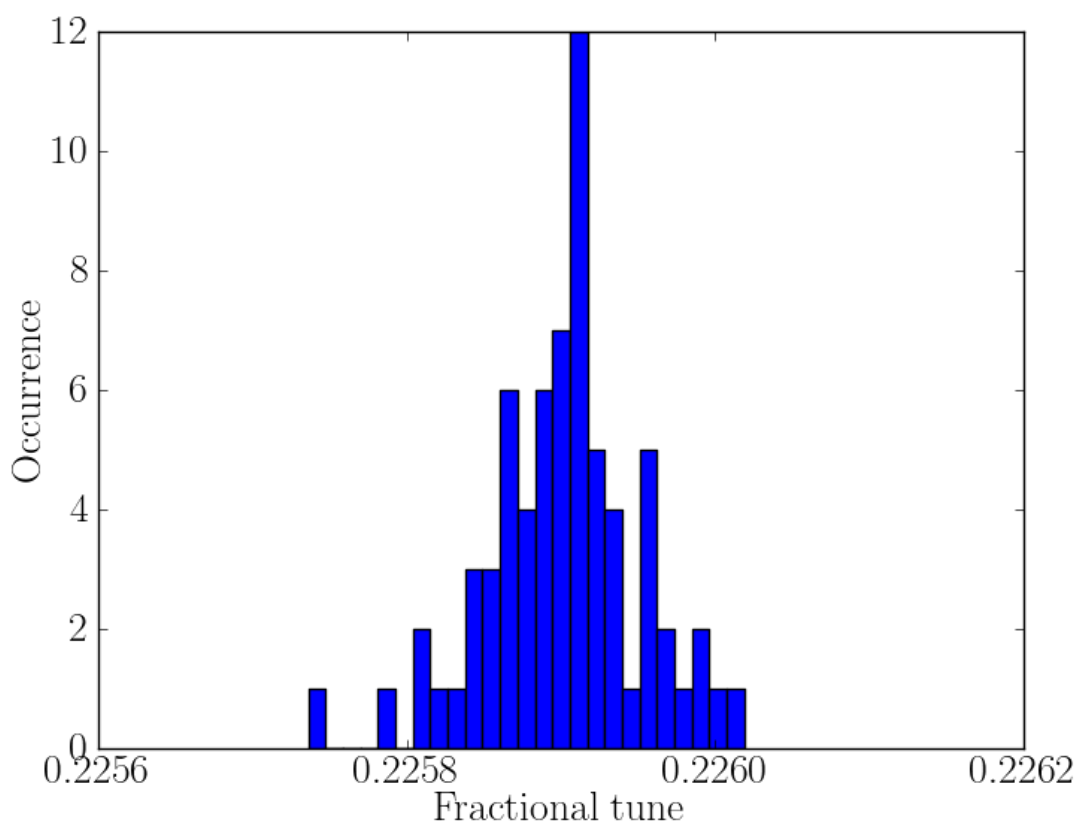

Figure 3: Histogram of the measured tunes (the RMS is $1.6 * 10^{-5}$ ). 


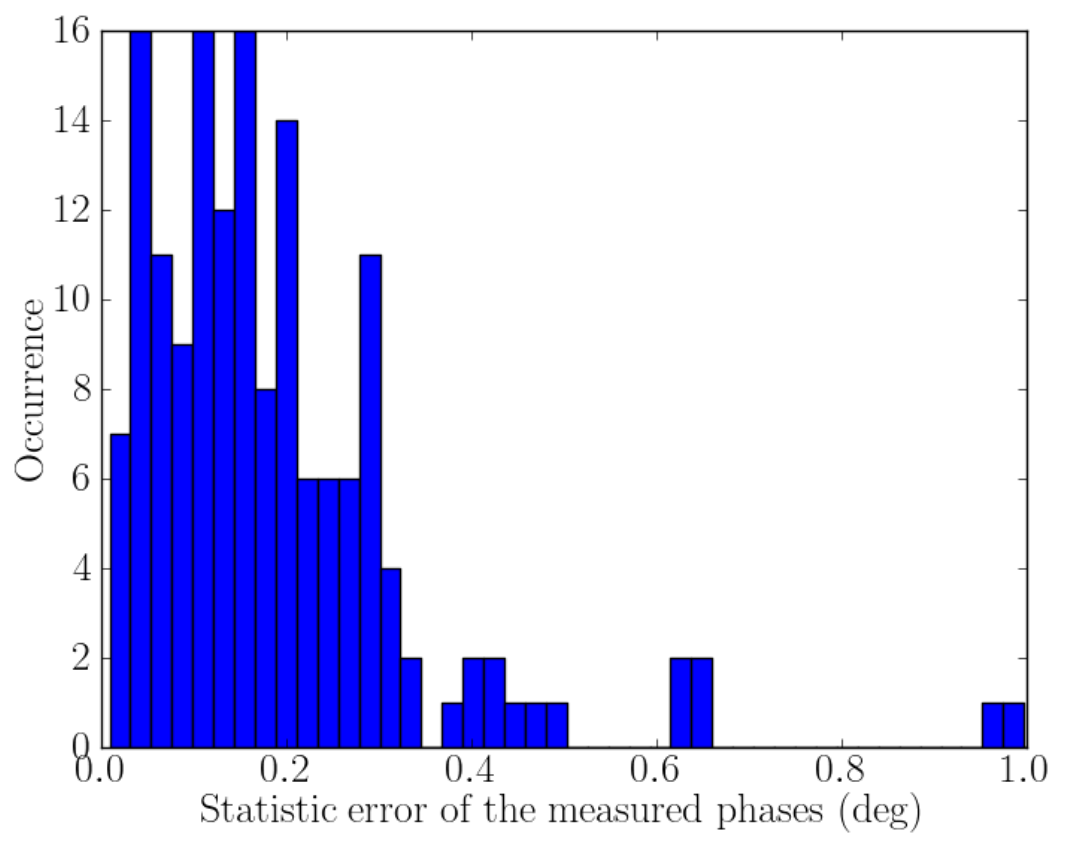

Figure 4: Statistic error of the measured phase advances.

was studied with four sets of turn-by-turn BPM data. The result is shown in Fig. 4.

The $\beta$-functions at BPMs can be calculated either using method in reference [16] or based on the amplitudes of the spectral peaks. Both methods rely on knowledge of the model optical functions. Due to the fact that the phase advance between most BPMs are close to $\pi / 2$, the latter method works better for $\beta$ function calculation in RHIC. In this article, linear optics measurement will be presented in the form of relative error of $\beta$-functions (beta-beat) since phase errors are correlated with $\beta$-function errors [17]. The statistic error of beta-beats is shown in Fig. 5.

\subsection{Acquiring turn-by-turn BPM data during acceleration}

The excitation of betatron oscillation during beam acceleration is unavoidably destructive, which causes beam loss and increase of beam emittances. The tune meter kicker is able to kick one bunch only so that minimize the impact on the beam. Turn-by-turn BPM data of free betatron oscillations in both planes can be recorded while kicking a bunch periodically on the ramp without deteriorating the transmission efficiency noticeably. The option of $1 \mathrm{k}, 2 \mathrm{k}$ and $4 \mathrm{k}$ data points in the turn-by-turn BPM data are available. The option of $1 \mathrm{k}$ is chosen for the ramp optics measurement since usually the oscillation lasts less than 500 turns due to decoherence. 


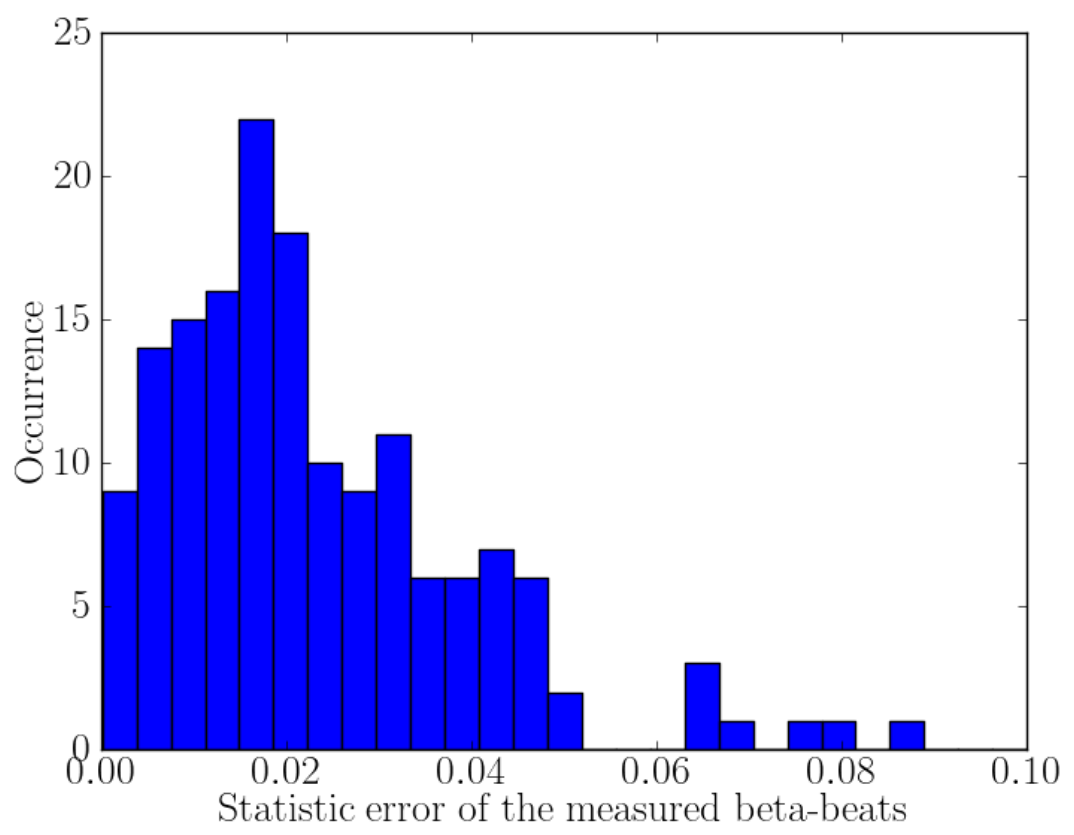

Figure 5: Statistic error of the measured relative error of $\beta$-functions. 
BLUE RING

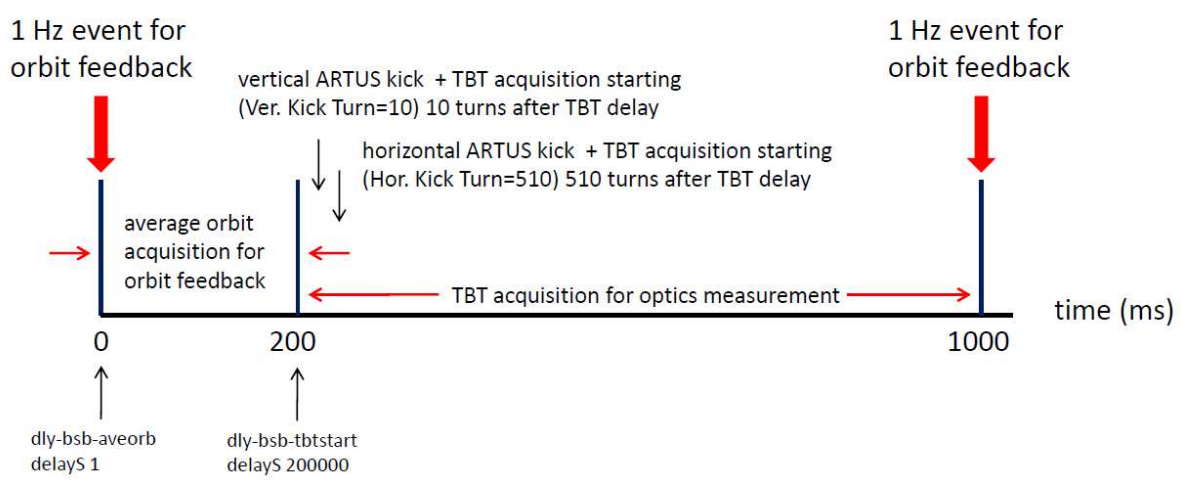

Figure 6: Conceptual sketch of carefully orchestrated timing to allow for turn-by-turn BPM acquisitions interleaved with BPM acquisitions required by orbit feedback during beam acceleration.

Measurements of the beam optics were made reproducible by ensuring reproducible beam orbits and betatron tunes using the now standard beam feedback systems during acceleration. While orbit and tune feedback operate independently, the BPM measurements used by orbit feedback and the turn-by-turn BPM measurements share the same networks for data delivery. The timing of the delivery of beam position measurements for these two systems was therefore carefully staggered to avoid data corruption. A conceptual sketch showing the data acquisition methodology is shown in Fig. 6 [12]. Orbit feedback operated at its standard $1 \mathrm{~Hz}$ rate. We allowed $200 \mathrm{~ms}$ corresponding to an upper limit on the time to transmit all (4 planes from both accelerators) the average orbit BPM data well in excess of the $150 \mathrm{~ms}$ required based on previous measurements [18]. After delivery of the data for orbit feedback, the beam was excited in one plane followed a short time later by excitation in the other plane, where the spacing between applied excitations was set ( $~ 500$ turns) to be longer than the decoherence time.

\subsection{Optics analysis results during beam acceleration}

With turn-by-turn BPM data acquired every $4 \mathrm{~s}$ during beam acceleration in both rings, we can collect typically $\sim 80$ sets of data during acceleration of beams to full energy for the Au-Au physics ramp in 2014 lasting 320 seconds total. As the ramp optics measurement happens as early as in the beam setup phase, the data may not be of good quality for retrieving reliable optical informations due to not yet optimized chromaticity settings. Some adjustments of the chromaticities during beam acceleration were necessary for improving beam transmission efficiency and the signal-to-noise ratio of the turn-by-turn data. 
Fortunately, the turn-by-turn data collected in a non-optimal beam condition could be used to direct us to adjust the chromaticities. This technique had been applied for ramp tuning in earlier years at RHIC [19]. The method has recently been revived since and has helped expedite the beam setup in 2014. With some iterations of ramp optics measurements and chromaticity adjustments, we acquired turn-by-turn BPM data of more than $\sim 200$ turns oscillation during the beam acceleration for further analysis of linear optics.

The model machine optics are required as well as the turn-by-turn orbit measurements for us to be able to analyze the linear optics information. The optics model during beam acceleration was retrieved from the RHIC on-line model [20] before-hand. In addition to the global beta-beat and phase errors, one can also determine the $\beta$-functions at locations of interest, like Ionization Profile Monitors (IPMs) and IPs.

Since the volume of data being acquired is large, we present the deviation of the machine optics in the form of global beta-beat Root-Mean-Square (RMS) during beam acceleration. Fig. 7 shows the RMS beta-beats at each time of optics measurement during beam acceleration for the $\mathrm{Au}-\mathrm{Au}$ physics ramp in 2014. The measurement error is missing in the plot because only one data set was acquired with the same optical settings. The statistical errors are expected to be larger than that shown in Fig. 5 because the chromaticities were optimized for beam transmission efficiency on the ramp rather than for high precision optics measurement. The measurements around transition at $\sim 85$ second were complicated by the difficulty of obtaining turn-by-turn beam position data. It was always the case that beta-beats of all planes in the midway of the beam acceleration are the minimum and start to grow as $\beta$-functions being squeezed in RHIC ramps.

The $\beta$-functions at the colliding IPs (6 and 8$)$ were also interpolated for the same set of measurement. The measured and model $\beta$-functions on the ramp are shown in Fig. 8 for the Blue ring only. The horizontal and vertical $\beta$-functions at IP6 are shown in the upper left and right plots, and the horizontal and vertical $\beta$-functions at IP8 are shown in the lower left and right plots. Despite the large measurement errors in the early part of the ramp, the measurements confirm the beta stars are squeezed as designed.

At the IPMs, model $\beta$-functions have been deployed for the calculation of emittances based on the measured beam sizes [21]. It is desirable to confirm whether the measured $\beta$-functions at the IPMs are consistent with the model values for better confidence in the emittance measurement. There are four IPM devices, one for each plane in both rings. The measured $\beta$-functions during acceleration are shown in Fig. 9 together with the model. As the $\beta$-functions were squeezed during acceleration, the deviation of the model and measured $\beta$-functions at the IPMs increased. The measured $\beta$-function reached $\sim 2$ times of the model value at the Yellow vertical IPM as shown in the lower right plot in Fig. 9. A larger measured $\beta$-function implies a reported beam emittance larger than reality as the reported emittance used the model $\beta$-function.

In 2013, the Yellow vertical IPM reported unphysical decreasing emittances in the final beta-squeeze on the special ramp for electron lens [22]. The mea- 

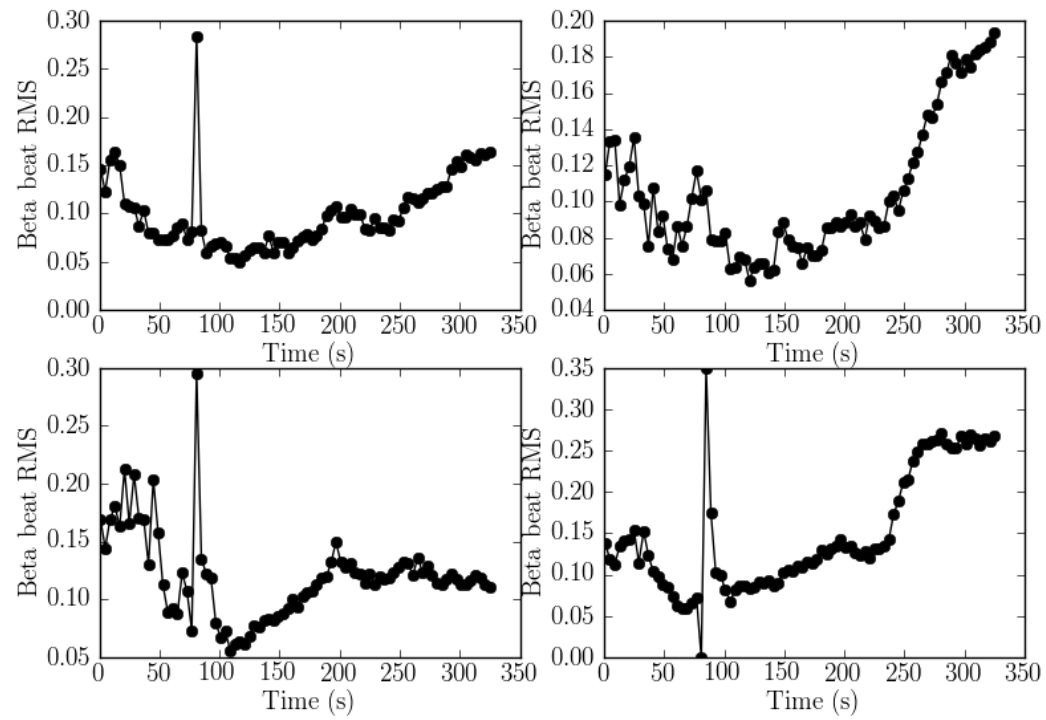

Figure 7: The measured beta-beats during beam acceleration (high energy $\mathrm{Au}-\mathrm{Au}, 2014$ ): the horizontal and vertical beta-beats RMS in the Blue ring are shown in the upper left and right plots, and the horizontal and vertical beta-beats RMS in the Yellow ring are shown in the lower left and right plots. 

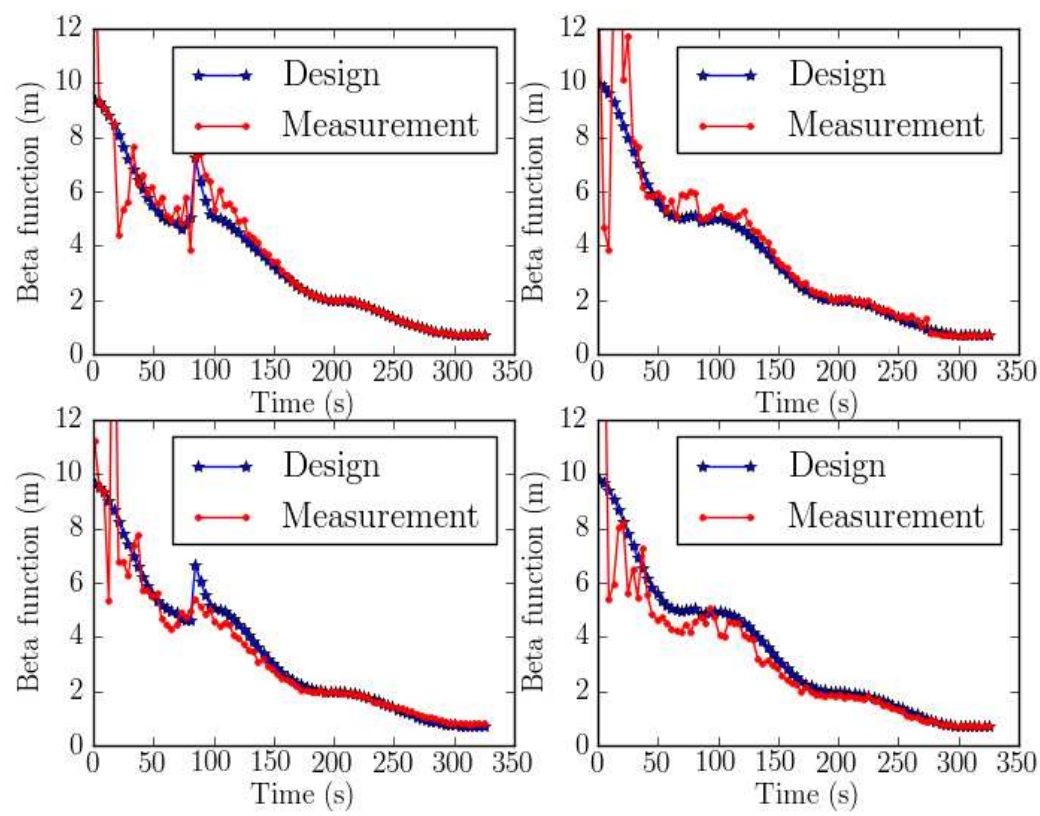

Figure 8: The measured and model beta stars at IP6 and IP8 (high energy Au-Au, 2014): the horizontal and vertical $\beta$-functions at IP6 in the Blue ring are shown in the upper left and right plots, and the horizontal and vertical $\beta$-functions at IP8 in the Blue ring are shown in the lower left and right plots. 

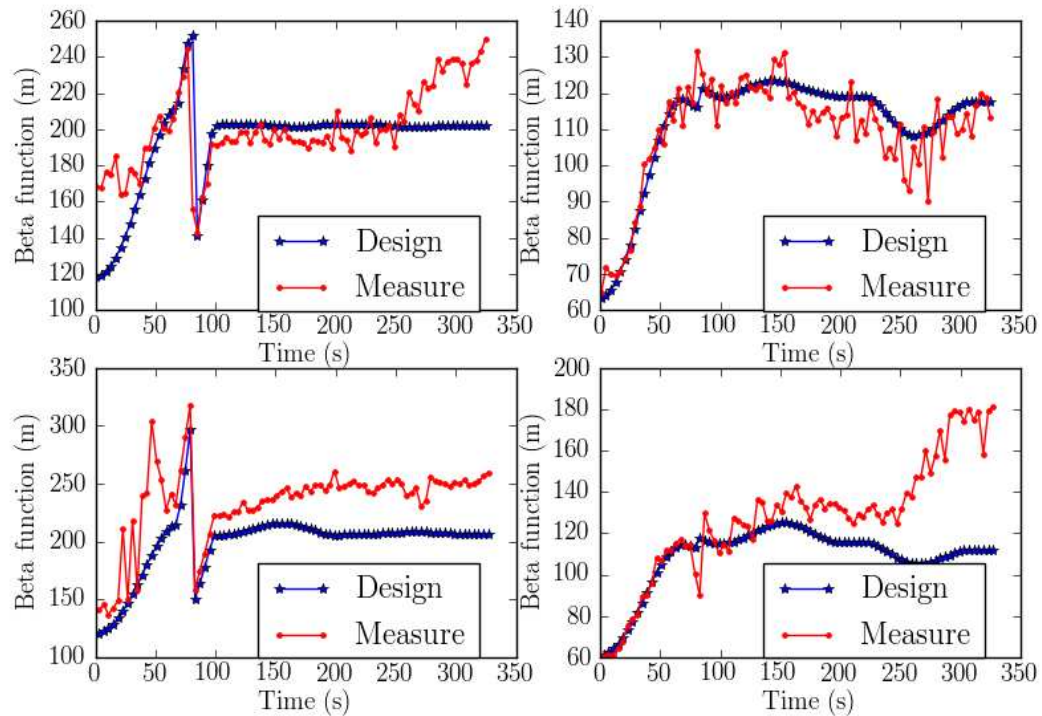

Figure 9: The measured and model $\beta$-functions at the IPMs (high energy $\mathrm{Au}-\mathrm{Au}, 2014$ ): the horizontal and vertical $\beta$-functions at the IPMs in the Blue ring are shown in the upper left and right plots, and the horizontal and vertical $\beta$-functions at the IPMs in the Yellow ring are shown in the lower left and right plots. Transition crossing occurs at $\sim 85$ seconds after the start of acceleration. 
sured beam size was more or less constant and the $\beta$-function at that IPM was designed to increase in that period. The $\beta$-functions interpolated from the ramp optics measurement revealed that the $\beta$-function at IPM was actually flat and decreasing in the late part [12]. The deviation of the measured $\beta$-functions from the model $\beta$-functions at the IPMs during acceleration revealed it is difficult to make judgment of the emittance increase on the ramp using the model $\beta$-functions in the presence of model-to-accelerator errors. One way of achieving a more precise emittance report is to apply the measured $\beta$-functions at the IPMs in the calculation of emittance on the ramp. The $\beta$-functions at the IPMs are interpolated for the entire ramp based on measurements at some fixed points to get the values at the time of the emittance measurements. The other way is to apply optics corrections on the ramp to minimize the discrepancy between the real machine optics and the model optics, which will be discussed further in the next section.

\section{Beam optics correction during acceleration}

The optics errors manifest themselves as beta-beat or phase advance errors. Both errors can be corrected by adjusting quadrupoles strengths based on a linear system model because their responses to quadrupole gradient changes are linear in the range of our consideration [23, 24, 25, 26, 27, 28, 29]. Correction of global beta-beat and phase errors has been demonstrated successfully with implementation for accelerator operations at RHIC in 2013 [30]. The basics of the two corrections being applied in RHIC are similar. Suppose $\left(e_{1}, e_{2}, \ldots, e_{m}\right)^{\prime}$ is the optics error (beta-beat or phase errors) being measured, $M$ is the response of the optics errors to quadrupole strength variations in the form of a matrix. The correction can be obtained by solving the following equations:

$$
-\left(\begin{array}{c}
e_{1} \\
e_{2} \\
\vdots \\
e_{m}
\end{array}\right)=\left(\begin{array}{cccc}
M_{11} & M_{12} & \cdots & M_{1 n} \\
M_{21} & M_{22} & \cdots & M_{2 n} \\
\vdots & \vdots & \ddots & \vdots \\
M_{m 1} & M_{m 2} & \cdots & M_{m n}
\end{array}\right) *\left(\begin{array}{c}
k_{1} \\
k_{2} \\
\vdots \\
k_{n}
\end{array}\right)
$$

The optics errors on the left side are from both planes, on which proper weights can be applied based on the scale of the errors. The correction knobs in RHIC are the 72 quadrupoles with trim power supplies which all reside in the interaction regions [1].

The response matrix for beta-beat correction can be calculated either by an optics program (like MAD-X) or analytically based on the on-line model optical functions. The analytical formula are presented in Eq. 4.

$$
\left\{\begin{array}{l}
M_{i j, x}=-\frac{\beta_{i, x}}{2 \sin \pi \mu_{x}} \cos \left(2 \pi \mu_{x}-2\left|\phi_{i, x}-\phi_{j, x}\right|\right) \\
M_{i j, y}=\frac{\beta_{i, y}}{2 \sin \pi \mu_{y}} \cos \left(2 \pi \mu_{y}-2\left|\phi_{i, y}-\phi_{j, y}\right|\right)
\end{array}\right.
$$

Betas in Eq. 4 are for the quadrupoles. The results from both calculations were compared for RHIC at both the injection and store energies. Because of the fact 
that the $\gamma$-transition quadrupoles [31] are implemented in the on-line model but not in the MAD-X model, the response matrices differ considerably from each other at injection energy [32]. At full energy, the two results agree very well with each other since the contribution of the $\gamma$-transition quadrupoles decreases with increasing energy. The response matrix for the phase error correction was only simulated with MAD-X. The error in the phase response matrix is expected to be non-negligible due to the missing $\gamma$-transition quadrupoles at injection. At full energy, the impact of the $\gamma$-transition quadrupoles on the phase response matrix should be minimal just like for the beta-beat response matrix.

\subsection{Optics correction at injection and store in RHIC}

At nominal injection energy, $\sim 23 \mathrm{GeV}$ for proton and $\sim 10 \mathrm{GeV}$ for gold beam, the optics errors were constantly monitored to be moderate. It was considered as necessary to correct the optics only if the peak-to-peak beta-beat exceeded $20 \%$ at injection. The optics correction is not expected to alleviate the emittance growth in the heavy ion case which is dominated by the intra-beam scattering, nor improve the lifetime for any species at injection.

In low energy runs in which the beam energy is lower than the nominal injection energy, the sextupole components in the dipole magnets can have a significant effect on the optics [33]. The optics error can be substantial enough so that applications based on model optics would not work properly. Furthermore, the deviation of beta stars at the colliding IPs should be corrected for better luminosity. These two reasons justify the necessity of optics corrections for low energy runs, as well as the potential of better beam lifetime. The turnby-turn BPM data from injection oscillation was used for optics analysis and correction [34]. The data acquisition was purely parasitic so that the optics was monitored for each physics store for the whole low energy run in 2014. This removed the burden of acquiring turn-by-turn BPM data by kicking the bunches whose intensity was on the low end limit for BPM monitoring. The corrections were applied in both rings. The beta-beat before and after the correction is shown in Fig. 10 for the Yellow ring only. The upper and lower plot show the horizontal and vertical beta-beat. The blue data points show the beta-beat before corrections and red points are from after corrections.

At store, BPM data for optics measurement was acquired by kicking the bunch with the tune meter kicker and recording the beam positions. The optics was usually measured when beams were not in collision to avoid beam-beam induced linear optics distortion. The principle of applying optics correction at store is the same as at injection. However, it is more desirable to have optics corrected at store for the direct benefit on the luminosity and luminosity lifetime. The results of optics correction at store will be presented in the following subsection together with the results of the optics correction during beam acceleration.

\subsection{Optics correction during beam acceleration}

In RHIC, the magnet ramping (or beam acceleration) is usually completed in two steps, called the energy ramp followed by the rotator ramp. During the 

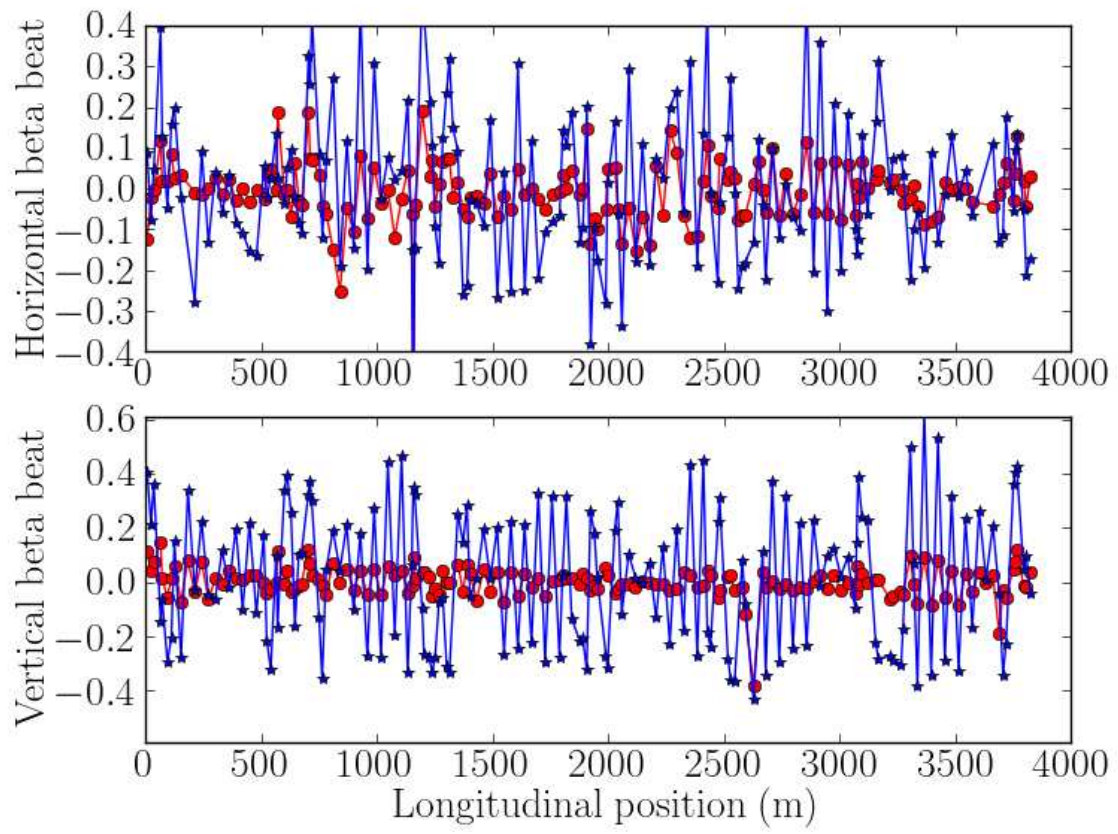

Figure 10: The beta-beats before and after optics correction in the Yellow ring for low energy run in 2014: the upper and lower plot show the horizontal and vertical beta-beats. The blue data point is the beta-beat before the corrections and red is after the corrections. 
energy ramp, the beam is accelerated to full energy and the $\beta$-functions are squeezed to the design values. For the polarized proton program, the currents of spin rotators are ramped up and the beam separation bumps collapse for collision in the rotator ramp. For the heavy ion program only the collapse of the separation bumps happens during the rotator ramp.

Compared to optics correction at a fixed energy, there are a number of unique challenges associated with correction of beam optics during beam acceleration. These include: (1) the ramping of the magnet power supplies can not be stopped and tuned, (2) large volume of corrections (typically 72 quadrupole power supplies at over 30 different times during the energy ramp) so that time-efficient implementation method is required, (3) consequences of the optics correction, such as change of tunes and chromaticities must be predicted and simultaneously compensated.

In the following, the tests on both the rotator ramp and the energy ramp will be presented which led to achieving optics corrections during beam acceleration.

\subsubsection{Optics correction during the rotator ramp}

First correction was applied during the fixed-energy rotator ramp for which the $\beta$-functions and phase advances are by design constant while the tunes and chromaticities vary as the separation bumps collapse. This was motivated by the large optical errors detected at the end of collision setup and by the desire to ensure minimal beam loss. The concerns regarding ramp optics correction implementation are mostly related to the beam loss and beam emittance dilution. As a test, correction on a rotator ramp with fewer bunches (12 by 12) was attempted to study these effects in 2013 proton program [35]. The test ramp was the same as a physics ramp except for the fewer bunches. The corrections calculated for the measured store optics were applied to all step-stones of the rotator ramp except the very first one. It means the correction strengths ramp up from the first to the second step-stone.

The results of the test eliminated the concern of adverse effects of ramp optics corrections. The beam loss was slightly better with optics correction implemented for the test ramp during the rotator ramp and after beam collision. The IPM reported emittance for the test ramp were compared with that for a physics ramp. They were similar except for the large error bars for the test ramp due to lower beam intensity with fewer bunches. In addition, the calculated beam emittance inferred from beam collision signals were as good as in a good physics store. The collision rate was expected to reach $1070 \mathrm{kHz}$ at STAR by scaling beam intensities to full machine ( $\sim 107$ bunches), which is $\sim 15 \%$ increase of luminosity compared with that for a nominal physics store. Later on, the correction for the rotator ramp was implemented operationally for the physics program in the Yellow ring only to avoid interference with other changes being made in the Blue ring. The rest of the 2013 run was executed with corrected optics without any complications. 


\subsubsection{Optics correction during the energy ramp}

Ramp optics correction for the energy ramp was tested twice in 2013 [35]. Ramp optics measurement was performed on a 12 bunches per accelerator ramp first. Then the turn-by-turn BPM data near the step-stones were selected for analysis of linear optics and calculation of optics corrections. The correction strengths at those step-stones were implemented through the RampEditor application. The problem encountered in the first attempt was that each time a set of strengths were sent to a step-stone in RampEditor, the on-line model recalculated all relevant magnet strengths for the whole ramp which was too time-consuming. The solution was to send all correction strengths for a stepstone once, not separately. The other way around is to hold constant (anchor) the magnet strengths for the relevant step-stones so interpolation of magnet strengths will not occur after implementing corrections. The two ways are similar because changing strengths for a step-stone would anchor it as well. The problem encountered in the second attempt was that the current curves for the magnets would change dramatically with step-stones settings being anchored, which caused power supplies to exceed their limits.

The difficulties of applying optics correction on the energy ramp were circumvented by a new strategy of implementation, proposed after the correction strength for all step-stones are examined. The calculated correction strength for all 72 trim quadrupoles in the Yellow ring on the energy ramp are displayed in Fig. 11. With the exception of the strength changes around transition crossing at around 85 second in the ramp, the required correction strength are linear between the dashed lines, which represent the step-stones where the settings for quadrupoles (some or all) are fixed. This prompted a strategy of implementing corrections only for the step-stones at the dashed lines, with correction strengths for every stones in-between dashed lines automatically interpolated. The change of strategy helped on reducing the number of corrections and avoiding the unnecessary anchoring of the quadrupoles strengths.

The new scheme of ramp optics correction was tested successfully without issues during the high energy Au-Au program in 2013. Six out of twenty three step-stones were picked for implementing optics corrections. They are "beta5", where beta stars are all squeezed down to $5 \mathrm{~m}$ and ready for transition crossing, "still5", where $\beta$-functions at colliding IPs continues squeezing after transition, "t200" and "t220", where beta stars at colliding IPs reach $2 \mathrm{~m}$, "flattop", where beam energy acceleration finishes, and "store". The global beta-beats before and after the corrections implemented are presented in Fig. 12 and 13 in terms of the RMS. The global beta-beats were not only greatly reduced at the vertical lines where corrections were implemented, but suppression of beta-beats of the same scale was achieved for anytime on the ramp where corrections were interpolated. Even though the benefit of optics correction around transition was not clear from linear optics measurement, the beam loss around transition was the lowest with corrections compared with that for the ramps in the same time period without corrections. It is worth noting that one needs to implement corrections for all quadrupoles at any step-stone with any of the 72 quadrupole settings anchored. 


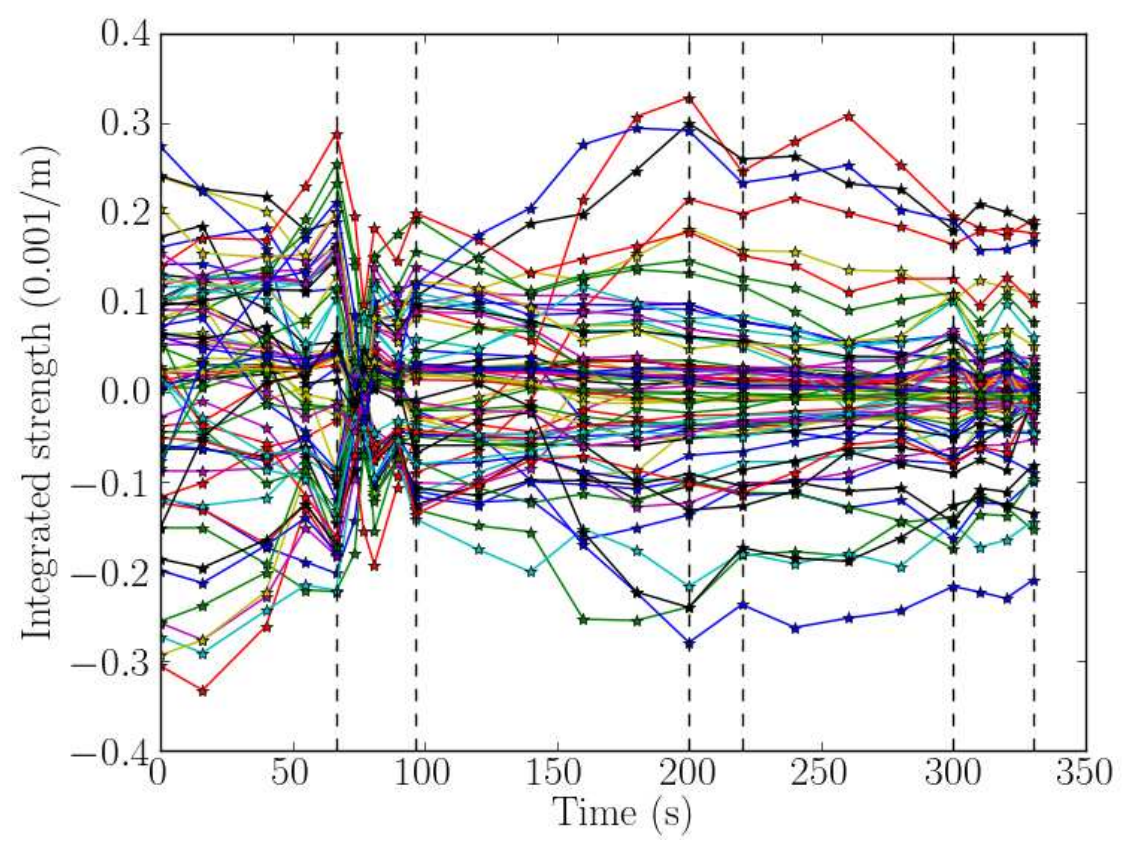

Figure 11: The calculated correction strength for the 72 trim quadrupoles in the Yellow ring on the energy ramp, the dashed lines are at the step-stones where settings for quadrupoles (some or all) are fixed. The horizontal axis is the time in seconds from the time the acceleration ramp starts.

Otherwise, the corrections will be ramped down to zero for the quadrupoles whose settings are anchored. For the first iteration, the correction was not applied at "t220" indicated by the solid vertical line, which resulted in a global beta-beat bump from "t200" to "flattop" with a peak at "t220". For the second iteration, the corrections were applied for the "t220" step-stones as well so that we eliminated the beta-beat bump and obtained the results shown in Fig. 12 and 13.

\subsection{Impact of optics correction during beam acceleration on emittance measure- ment}

As mentioned before, the $\beta$-functions at the IPMs on the ramp are of particular interest since they are the crucial tool for monitoring the emittance during beam acceleration. The $\beta$-functions before and after implementing optics correction on the ramp are shown in Fig. 14 and 15 together with the designed values. The agreement between the measured $\beta$-functions and the design values were much improved with optics correction during acceleration implemented. This facilitates a much more precise characterization of the emittance on the ramp. 


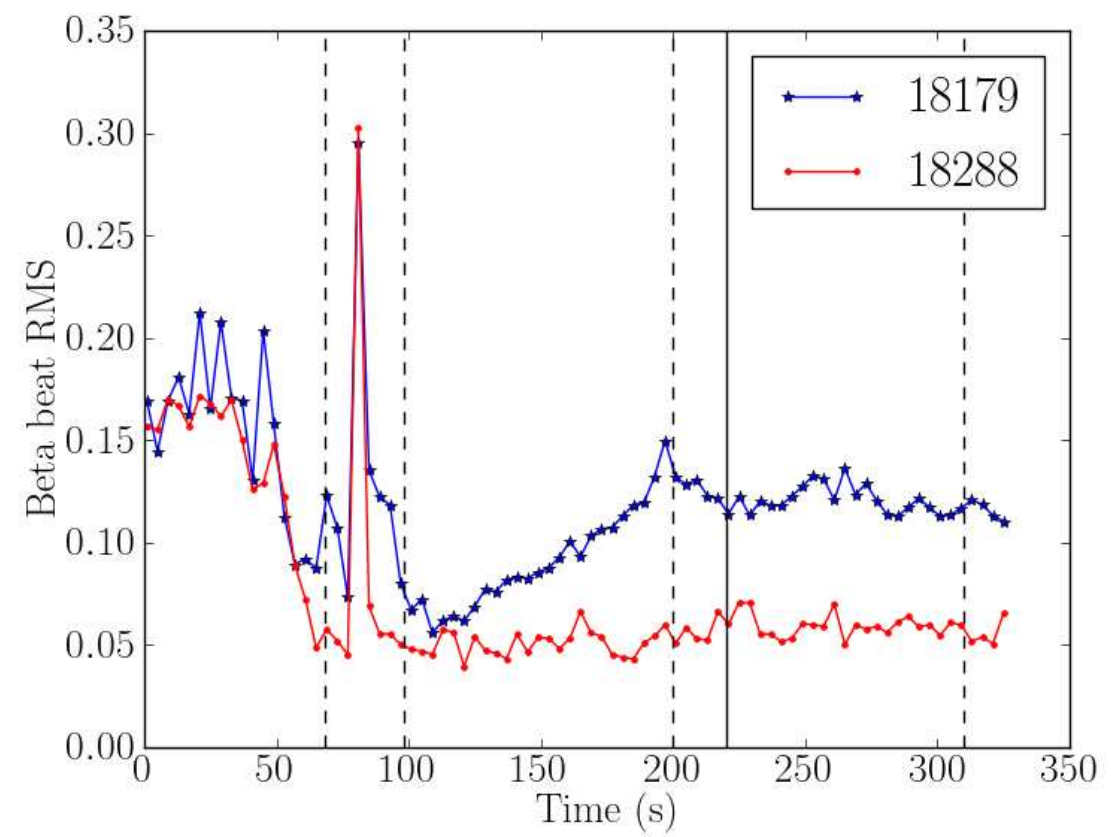

Figure 12: The global RMS beta-beats in the Yellow ring horizontal plane measured for two fills, 18179 and 18288, optics corrections were implemented for the second fill at 6 out of 23 step-stones, at times indicated by the vertical lines with linear interpolation in-between.

With optics correction being implemented during beam acceleration, the reported beam emittance by IPMs based on design $\beta$-functions were more reasonable. With stochastic cooling, the beam emittance at full energy for both planes in each ring reduced to agree with each other, which indicates accurate emittance measurements [36]. The emittance reported by IPMs and deduced from collision rates agreed with each other for the first time in RHIC, using measured $\beta$-functions at corresponding locations [36]. The future plan for an even more accurate emittance measurement during beam acceleration is to implement optics correction during beam acceleration first, then obtain measured $\beta$-functions at the IPMs by ramp optics measurement and apply these for emittance calculations. This scheme can further reduce the systematic error in emittance measurement on the ramp due to residual beta-beats.

\section{Summary}

The optics measurement during beam acceleration in RHIC was first demonstrated and implemented during operations in 2013. The measurement results have been used to find abnormality of the ramp (for example, unphysical emittance change on the ramp), determine gradient errors and corrections, interpo- 


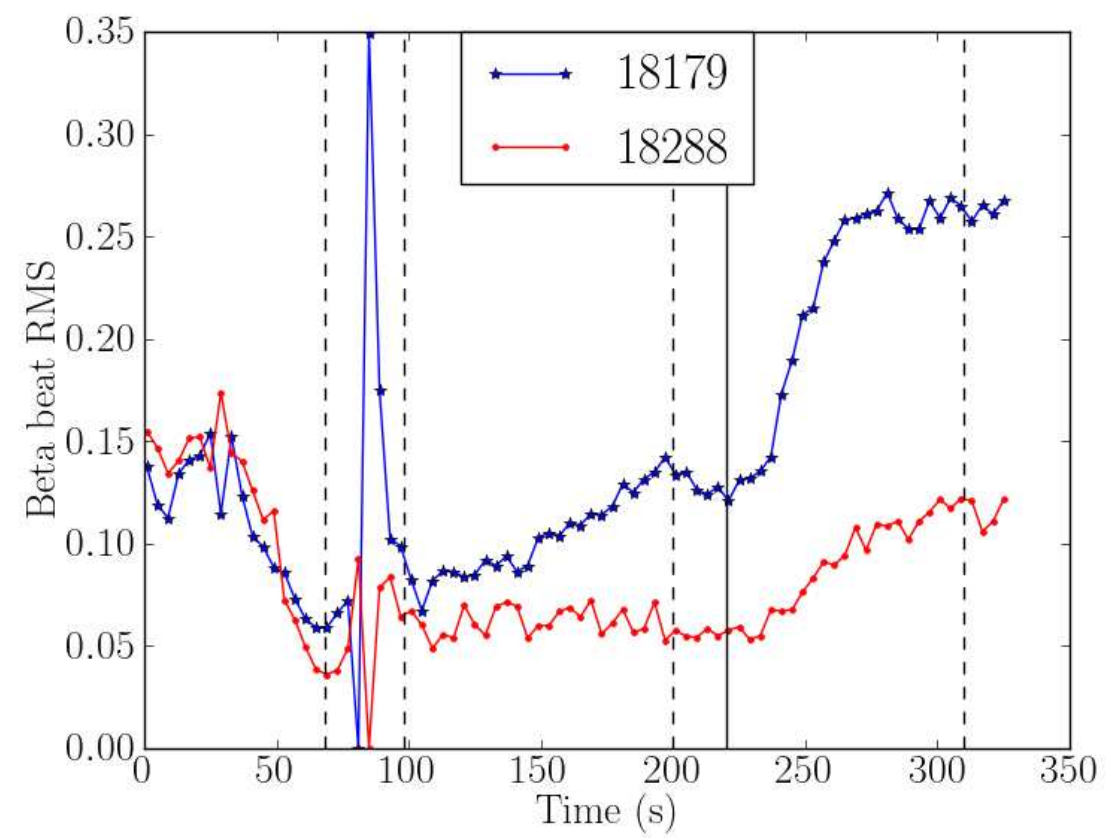

Figure 13: The global RMS beta-beats in the Yellow ring vertical plane measured for two fills, 18179 and 18288, optics corrections were implemented for the second fill at 6 out of 23 step-stones, at times indicated by the vertical lines with linear interpolation in-between.

late the measured optical functions to intermediate locations (e.g. at IPs, IPMs, polarimeters, Schottky detectors) and facilitate the tuning of the acceleration ramp. The difficulties encountered when implementing optics correction during beam acceleration in 2013 was overcome after measurements revealed that applying corrections for selected step-stones would be sufficient. The interpolated corrections for any point in-between those selected step-stones worked well for the ramp. The optics correction during beam acceleration was implemented operationally for high energy $\mathrm{Au}-\mathrm{Au}$ and $\mathrm{He} 3-\mathrm{Au}$ physics programs in 2014. The beta-beats was reduced substantially on the ramp for the first time in a hadron collider by the optics correction during beam acceleration. The milestones in the development of ramp optics measurement and correction during beam acceleration in RHIC are given in Appendix A. The errors in reported emittance by the IPMs due to imperfect model of the $\beta$-functions were significantly reduced with corrected optics.

\section{Acknowledgment}

We would like to thank A. Drees, R. Michnoff, I. Blackler and G. Marr's contributions of implementing ramp optics measurement in the tape sequence, 


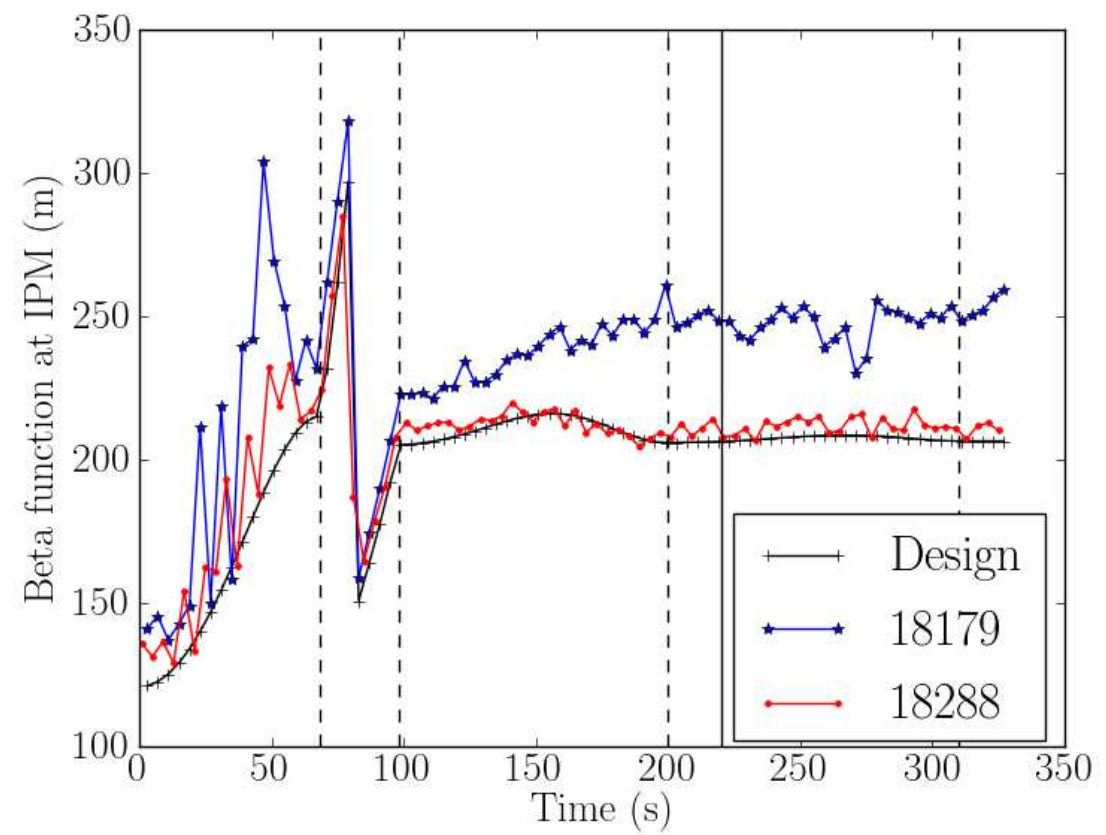

Figure 14: The measured $\beta$-functions on the ramp at the horizontal IPM in the Yellow ring for two fills, 18179 and 18288, the black plus signs are the design values, the blue stars are the measured $\beta$-functions for fill 18179 , the red dots are the $\beta$-functions measured for fill 18288 for which the ramp optics corrections were implemented.

W. Fischer, M. Blaskiewicz, G. Robert-Demolaize (run coordinator for high energy Au-Au program in 2014) and the operation crews for the encouragement and support of our work, also V. Schoefer, G. Marr and T. Shrey for their support of our work during the startup phase.

[1] H. Hahn. RHIC design manual, October 2000.

[2] J. Van Zeijts and A. Marusic. Ramp design and implementation for the RHIC Deuteron-Gold and polarized proton runs. Proceedings of the ICALEPCS 2003.

[3] J. van Zeijts. Model driven ramp control at RHIC. arXiv preprint physics/0111066, 2001.

[4] M. Minty, A. Marusic, A. Curcio, C. Dawson, C. Degen, W. Fischer, R. Hulsart, Y. Luo, G. Marr, K. Mernick, et al. Simultaneous orbit, tune, coupling and chromaticity feedback at RHIC. Proceedings of the PAC 2011.

[5] P. Thieberger. Private communication. 


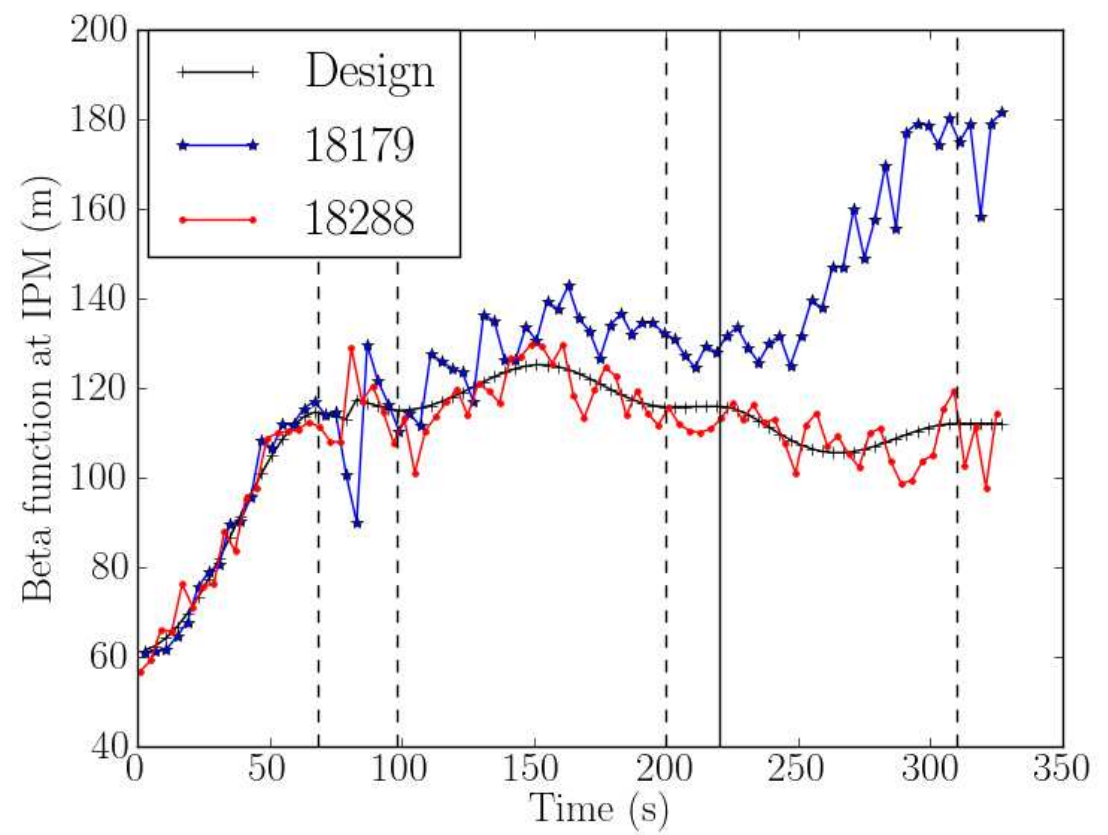

Figure 15: The measured $\beta$-functions on the ramp at the vertical IPM in the Yellow ring for two fills, 18179 and 18288 , the black plus signs are the design values, the blue stars are the measured $\beta$-functions for fill 18179 , the red dots are the $\beta$-functions measured for fill 18288 for which the ramp optics corrections were implemented.

[6] R. Bartolini, M. Giovannozzi, W. Scandale, A. Bazzani, and E. Todesco. Algorithms for a precise determination of the betatron tune. Proceedings of the EPAC 1996.

[7] G. Vanbavinckhove, M. Aiba, A. Nadji, L. Nadolski, R. Tomás, and MA Tordeux. Linear and non-linear optics measurements at SOLEIL. Proceedings of the PAC 2009.

[8] M. Aiba, S. Fartoukh, A. Franchi, M. Giovannozzi, V. Kain, M. Lamont, R. Tomás, G. Vanbavinckhove, J. Wenninger, F. Zimmermann, et al. First $\beta$-beating measurement and optics analysis for the CERN Large Hadron Collider. Physical Review Special Topics-Accelerators and Beams, 12(8):081002, 2009.

[9] YT Yan and Y. Cai. Precision PEP-II optics measurement with an SVD-enhanced Least-Square fitting. Nuclear Instruments and Methods in Physics Research Section A: Accelerators, Spectrometers, Detectors and Associated Equipment, 558(1):336-339, 2006.

[10] X. Huang, SY Lee, E. Prebys, and R. Tomlin. Application of independent 
component analysis to Fermilab booster. Physical Review Special TopicsAccelerators and Beams, 8(6):064001, 2005.

[11] C. Liu, R. Hulsart, A. Marusic, M. Minty, R. Michnoff, P. Thieberger, et al. Precision tune, phase and beta function measurement by frequency analysis in RHIC. Proceedings of the IPAC 2013.

[12] M. Minty, KA Drees, R. Hulsart, C. Liu, A. Marusic, R. Michnoff, and P. Thieberger. Measurement of beam optics during acceleration in the Relativistic Heavy Ion Collider. Proceedings of the NA-PAC 2013.

[13] Fusheng Zhang, Zhongxing Geng, and Wei Yuan. The algorithm of interpolating windowed FFT for harmonic analysis of electric power system. Power Delivery, IEEE Transactions on, 16(2):160-164, 2001.

[14] M. Gasior and JL Gonzalez. Improving FFT frequency measurement resoulution by parabolic and Gaussian spectrum interpolation. AIP Conf. Proc., 732(CERN-AB-2004-023-BDI):276-285, 2004.

[15] CSS Burrus and Thomas W Parks. DFT/FFT and Convolution Algorithms: Theory and Implementation. John Wiley \& Sons, Inc., 1991.

[16] P. Castro, J. Borer, A. Burns, G. Morpurgo, and R. Schmidt. Betatron function measurement at LEP using the BOM 1000 turns facility. Proceedings of the PAC 1993.

[17] R. Tomás, Y. Papaphilippou, S. Fartoukh, F. Zimmermann, R. Calaga, A. Franchi, M. Giovannozzi, O. Brüning, and S. Peggs. Procedures and accuracy estimates for beta-beat correction in the LHC. Technical report, LHC-PROJECT-Report-941, 2006.

[18] M. Minty, R. Hulsart, A. Marusic, R. Michnoff, V. Ptitsyn, G. RobertDemolaize, and T. Satogata. Global orbit feedback at RHIC. Proceedings of the IPAC 2010.

[19] D. Trbojevic, V. Ptitsyn, W. Fischer, L. Ahrens, M. Blaskiewicz, T. Hayes, F. Pilat, T. Roser, S. Tepikian, P. Cameron, et al. Improvements of the RHIC ramp efficiency. Proceedings of EPAC 2002.

[20] N. Malitsky, K. Brown, N. D’Imperio, J. Kewisch, A. Fedotov, A. Luccio, F. Pilat, V. Ptitsyn, T. Satogata, S. Tepikian, et al. Joining the RHIC online and off-line models. Proceedings of the PAC 2005.

[21] R. Connolly, R. Michnoff, T. Moore, T. Shea, and S. Tepikian. Beam profile measurements and transverse phase-space reconstruction on the relativistic heavy ion collider. Nuclear Instruments and Methods in Physics Research Section A: Accelerators, Spectrometers, Detectors and Associated Equipment, 443(2):215-222, 2000. 
[22] X. Gu, Z. Altinbas, M. Anerella, D. Bruno, M. Costanzo, WC Dawson, AK Drees, W. Fischer, M. Frak, DM Gassner, et al. Commissioning RHICs electron lens. Proceedings of the NA-PAC 2013.

[23] R. Tomás, O. Brüning, M. Giovannozzi, P. Hagen, M. Lamont, F. Schmidt, G. Vanbavinckhove, M. Aiba, R. Calaga, and R. Miyamoto. CERN Large Hadron Collider optics model, measurements, and corrections. Physical Review Special Topics-Accelerators And Beams, 13(12):121004, 2010.

[24] V. Lebedev, V. Nagaslaev, A. Valishev, and V. Sajaev. Measurement and correction of linear optics and coupling at tevatron complex. Nuclear Instruments and Methods in Physics Research Section A: Accelerators, Spectrometers, Detectors and Associated Equipment, 558(1):299-302, 2006.

[25] G. Vanbavinckhove, M. Bai, and G. Robert-Demolaize. Optics corrections at RHIC. Proceedings of the IPAC 2011.

[26] G. Wang, M. Bai, and L. Yang. Linear optics measurements and corrections using AC dipole in RHIC. Proceedings of the IPAC 2010.

[27] M. Bai, J. Aronson, M. Blaskiewicz, Y. Luo, G. Robert-Demolaize, S. White, and G. Vanbavinckhove. Optics measurements and corrections at RHIC. Proceedings of the IPAC 2012.

[28] R. Calaga. Linear beam dynamics and ampere class superconducting RF cavities@RHIC.PhD thesis, Stony Brook University, 2006.

[29] X. Shen, G. Robert-Domolaize, S. White, Y. Luo, A. Marusic, M. Bai, SY Lee, and R. Tomás. Ac dipole based optics measurement and correction at RHIC. Proceedings of the NA-PAC 2013.

[30] C. Liu, M. Blaskiewicz, KA Drees, A. Marusic, and M. Minty. Global optics correction in rhic based on turn-by-turn data from the ARTUS tune meter. Proceedings of the NA-PAC 2013.

[31] C. Montag and J. Kewisch. Commissioning of a first-order matched transition jump at the Brookhaven Relativistic Heavy Ion Collider. Physical Review Special Topics-Accelerators and Beams, 7(1):011001, 2004.

[32] S. Tepikian. Private communication.

[33] C. Montag. Multipole error data analysis for RHIC low-energy operations. Technical report, C-A/AP/421, 2011.

[34] C. Liu, K.A. Drees, A. Marusic, M. Minty, and C. Montag. Global optics correction for low energy RHIC run, To be published.

[35] C. Liu, A. Marusic, and M. Minty. Implementation of optics correction on the ramp in RHIC. Proceedings of the NA-PAC 2013. 
[36] M. Minty, R. Connolly, C. Liu, T. Summers, and S. Tepikian. Absolute beam emittance measurements at RHIC using ionization profile monitors. Technical report, C-A/AP/522.

Appendix A. Milestones in the development of ramp optics measurement and correction during beam acceleration in RHIC 
Table A.1: Milestones in the development of ramp optics measurement and correction during beam acceleration in RHIC.

\begin{tabular}{|c|c|c|c|c|}
\hline Fill & Date & Ramp & Test & Result \\
\hline 17185 & 03/07/13 & pp13e-v4/s4 & $\begin{array}{l}\text { Ramp optics } \\
\text { measurement }\end{array}$ & $\begin{array}{l}\text { First demonstration of ac- } \\
\text { quisition of multiple sets } \\
\text { of turn-by-turn data from } \\
\text { all BPMs during accelera- } \\
\text { tion }\end{array}$ \\
\hline 17245 & $03 / 18 / 13$ & pp13e-s4 & $\begin{array}{l}\text { Ramp optics } \\
\text { measurement }\end{array}$ & $\begin{array}{l}\text { Full data sets acquired si- } \\
\text { multaneously in both the } \\
\text { blue and yellow rings dur- } \\
\text { ing acceleration }\end{array}$ \\
\hline 17354 & $04 / 10 / 13$ & pp13b-v2 & $\begin{array}{l}\text { Ramp optics } \\
\text { measurement }\end{array}$ & $\begin{array}{l}\text { Repeat measurements for } \\
\text { both beams during accel- } \\
\text { eration }\end{array}$ \\
\hline 17451 & $05 / 02 / 13$ & pp13b-v2 & $\begin{array}{l}\text { Ramp optics } \\
\text { correction }\end{array}$ & $\begin{array}{l}\text { First implementation of } \\
\text { correction in operation } \\
\text { during the rotator ramp }\end{array}$ \\
\hline 18170 & $04 / 04 / 13$ & Au14-v0 & $\begin{array}{l}\text { Ramp optics } \\
\text { measurement }\end{array}$ & $\begin{array}{l}\text { Test of sequencing for au- } \\
\text { tomation of ramp optics } \\
\text { measurement }\end{array}$ \\
\hline 18179 & $04 / 08 / 14$ & Au14-v0 & $\begin{array}{l}\text { Ramp optics } \\
\text { measurement }\end{array}$ & $\begin{array}{l}\text { First ramp optics mea- } \\
\text { surement by automated } \\
\text { tape sequence }\end{array}$ \\
\hline 18238 & $04 / 23 / 14$ & Au14-v0 & $\begin{array}{l}\text { Ramp optics } \\
\text { correction }\end{array}$ & $\begin{array}{l}\text { First demonstration of } \\
\text { correction during the } \\
\text { energy ramp in the Yellow } \\
\text { ring }\end{array}$ \\
\hline 18288 & $05 / 07 / 14$ & Au14-v0 & $\begin{array}{l}\text { Ramp optics } \\
\text { correction }\end{array}$ & $\begin{array}{l}\text { First demonstration of } \\
\text { correction during the } \\
\text { energy ramp in the Blue } \\
\text { ring }\end{array}$ \\
\hline 18337 & $05 / 21 / 14$ & $\mathrm{Au} 14-\mathrm{v} 0 / \mathrm{s} 0$ & $\begin{array}{l}\text { Ramp optics } \\
\text { correction }\end{array}$ & $\begin{array}{l}\text { Chromaticity tuning in } \\
\text { the Yellow ring at Au14- } \\
\text { s0::store }\end{array}$ \\
\hline $18349 / 50$ & $05 / 23 / 14$ & Au14-v0 & $\begin{array}{l}\text { Ramp optics } \\
\text { correction }\end{array}$ & $\begin{array}{l}\text { Test of correction for } \\
\text { physics ramp }\end{array}$ \\
\hline 18397 & $06 / 06 / 14$ & Au14-v0 & $\begin{array}{l}\text { Ramp optics } \\
\text { correction }\end{array}$ & $\begin{array}{l}\text { Implemented in operation } \\
\text { for high energy } \mathrm{Au}-\mathrm{Au} \\
\text { program }\end{array}$ \\
\hline 18447 & $06 / 18 / 14$ & He3Au14-e0/s0 & $\begin{array}{l}\text { Ramp optics } \\
\text { correction }\end{array}$ & $\begin{array}{l}\text { Implemented in operation } \\
\text { for He3-Au program }\end{array}$ \\
\hline
\end{tabular}

